\title{
ATENCIÓN DEL PARTO CON FETO EN PRESENTACIÓN PELVIANA: REVISIÓN DE LA SEMIOLOGÍA, EL MECANISMO Y LA ATENCIÓN DEL PARTO
}

\section{Breech presentation delivery care: A review of chilldbinth semiology, mechanism and care}

Carlos Fernando Grillo-Ardila ${ }^{1}, M D, M S c$; Alejandro Antonio Bautista-Charry ${ }^{2}$, MD, MS(c); Mariana Diosa-Restrepo ${ }^{3}$, MD

Recibido: 25 de marzo de 2019/ Aceptado: 27 de diciembre de 2019

\section{RESUMEN}

Objetivo: revisar los conceptos que subyacen al trabajo de parto con feto en presentación pelviana, su semiología y las maniobras obstétricas que facilitan un resultado materno perinatal exitoso.

Materiales y métodos: a partir de un caso hipotético que ambienta de manera práctica el tema, se crea un documento explicativo construido a partir de una revisión narrativa, en donde se examinan los preceptos relacionados con el diagnóstico, el mecanismo del parto en presentación pelviana y el manejo intraparto de la gestante, con énfasis en la adecuada ejecución de las maniobras que facilitan la extracción fetal.

* Correspondencia: Carlos Fernando Grillo-Ardila, Departamento de Obstetricia y Ginecología, Universidad Nacional de Colombia, Carrera 30 No. 45-03, Edificio 471, Oficina 205, Bogotá, Colombia. Tel.: +5713165000 extensión 15122. cfgrilloa@unal.edu.co

1 Profesor asistente: docente de cátedra, Departamento de Obstetricia y Ginecología, Universidad Nacional de Colombia, Bogotá, Colombia.

2 Profesor Asociado; director del Departamento de Obstetricia y Ginecología, Universidad Nacional de Colombia, Bogotá, Colombia.

3 Residente de tercer año de Obstetricia y Ginecología, Facultad de Medicina, Universidad Nacional de Colombia, Bogotá, Colombia.
Resultados: el parto en presentación pelviana debe ser atendido por vía vaginal cuando se encuentra en periodo expulsivo con encajamiento cumplido. Para su diagnóstico y atención es esencial hacer una adecuada identificación de la presentación, así como conocer su fisiología, la indicación y adecuada ejecución de las maniobras obstétricas que facilitan un parto sin complicaciones.

Conclusiones: el mecanismo del parto en presentación pelviana es complejo y requiere, cuando no hay otra alternativa para la atención, que tanto el obstetra como el médico general conozcan su fisiología y las múltiples maniobras obstétricas que facilitan obtener buen resultado materno perinatal. Palabras clave: presentación pelviana; complicaciones obstétricas del parto; educación médica continuada; distocia.

\section{ABSTRACT}

Objective: To review the concepts underlying breech presentation delivery as well as the semiology and the obstetric maneuvers contributing to a successful perinatal maternal outcome. 
Materials and methods: Based on a hypothetical scenario to set the stage for a practical approach to the topic, an explanatory paper built on a narrative review is created in order to examine the principles related to diagnosis, mechanism of delivery and maternal care, emphasizing maneuvers to ease fetal extraction.

Results: Breech presentation delivery must be managed through the vaginal canal when already in the expulsion phase with fetal engagement. For diagnosis and care, it is essential to know the unique semiology and physiology of this condition as well as the obstetric maneuvers to facilitate an uncomplicated delivery.

Conclusions: The mechanism of childbirth in breech presentation is complex and requires knowledge of its physiology and multiple obstetric maneuvers on the part of the obstetrician as well as the general practitioner, in order to ensure adequate care when there is no other option.

Key words: Breech presentation; obstetric complications of childbirth; continuing medical education; dystocia.

\section{INTRODUCCIÓN}

La presentación pelviana es aquella en la que el polo pélvico o caudal del feto está en relación directa con el estrecho superior de la pelvis materna, lo ocupa en su totalidad y sigue un mecanismo de parto conocido (1). Se consideran tres modalidades para esta presentación: la pelvis completa, modalidad franca de nalgas y pelvis incompleta (2).

La pelvis completa (pelviana completa) ocurre cuando los muslos fetales se encuentran flexionados sobre el abdomen y, a su vez, las piernas están sobre los muslos. En esta modalidad se mantiene la actitud fetal de flexión en todos los polos del feto (3). Por su parte, la modalidad franca de nalgas (pelviana simple) se presenta cuando los muslos se encuentran flexionados sobre el abdomen fetal pero las piernas están extendidas; representa la más frecuente de las tres modalidades, con el $64 \%$ de los casos (4). Finalmente, la pelvis incompleta (presentación parcial) ocurre cuando uno o ambos pies se ubican como la parte más avanzada de la presentación (1); el feto se encuentra literalmente parado en el canal del parto y representa una verdadera presentación de pies. La escuela española no acepta la presencia de esta modalidad, más allá de considerarla transitoria hacia una de las dos anteriores (3). Esta modalidad es considerada como la de peor pronóstico hacia la evolución espontánea del nacimiento, por la frecuente asociación con deflexión de los brazos a nivel de los hombros fetales (extensión de brazos nucales) y deflexión del polo cefálico (3).

Acorde con la literatura, se estima que el $20 \%$ de los fetos con edad gestacional menor a 28 semanas se encuentran en presentación pelviana (5), de los cuales el $4 \%$ permanecerá en esta postura más allá de la semana 36 (6); de esta forma, esta presentación solo debe ser considerada anómala hasta el término (1). A lo largo de la gestación, el feto debe realizar la "versión fetal", que es un fenómeno activo, en donde el fruto sano asume la posición de "mejor ajuste" siguiendo los principios clásicos de las leyes de Pajot (5). Por tanto, el hallazgo de un feto en presentación pelviana al final de la gestación, tácitamente conlleva la búsqueda de su factor desencadenante $(5,7)$, que puede tener su origen en la presencia de patologías de origen materno $(1,2)$ o fetal, dentro de las cuales sobresale la presencia de anomalías fetales o aneuploidías, alteraciones de la dinámica del líquido amniótico, perturbaciones en el canal del parto, placentación anómala (8) o la muerte fetal, entre otros $(1,2,5)$. Es importante mencionar que la frecuencia de anomalía fetal mayor es del $17 \%$ en los nacimientos prematuros en presentación de nalgas, del $9 \%$ en presentación de nalgas a término y del $50 \%$ en los neonatos que nacen con esta presentación y mueren (9).

Los frutos en presentación pelviana tienen mayor morbimortalidad cuando se comparan con aquellos que se presentan cefálicos, incluso si el nacimiento se produce por cesárea (5). Múltiples estudios han vinculado la presentación pelviana con aumento en el riesgo de muerte neonatal (Riesgo Relativo 
$[\mathrm{RR}]=2,3$; Intervalo de Confianza [IC] $95 \%: 2,1-$ $2,6)(10)$ o fetal $(\mathrm{RR}=12,52$; IC $95 \%: 7,86-9,95)$ (11), parto prolongado (RR = 8,05; IC $95 \%$ : 3,0011,47), asfixia ( $R R=10,24$; IC $95 \%: 4,92-21,31)$ (12), trauma (RR = 9,9; IC $95 \%: 1,8-55,6)(13)$ o bajo APGAR (RR = 2,4; IC 95\%: 1,1-4,6) (14). Resultados que ratifican esta presentación, per se, como un marcador de pobre pronóstico $(7,15)$.

Cuando nos remitimos a la práctica clínica, el escenario tampoco es alentador. Un estudio reciente demuestra que difícilmente el $15 \%$ de los obstetras se sienten confiados en la atención del parto en pelvis por vía vaginal, y que tan solo el $32 \%$ se encuentran capacitados para resolver esta condición clínica $(16,17)$. Ante este escenario, muchas personas recurren a la cesárea universal, por el limitado nivel de confianza, la ansiedad que rodea este proceso, los miedos médico-legales y los retos que impone esta situación $(18,19)$.

Ocasionalmente, en la práctica clínica encontramos pacientes con fetos en presentación de pelvis, que se encuentran con encajamiento y descenso avanzado en el canal del parto. Por tanto, el objetivo de este documento es revisar los conceptos que subyacen al trabajo de parto con feto en presentación pelviana, así como también la semiología y las maniobras obstétricas que facilitan un resultado materno perinatal exitoso.

\section{MATERIALES Y MÉTODOS}

A partir del caso hipotético presentado, se crea un documento explicativo en el que se revisan de manera narrativa los conceptos relacionados con el diagnóstico, mecanismo de parto y cuidado de la gestante, finalizando con las maniobras que facilitan la extracción fetal.

\section{ESCENARIO CLÍNICO}

Paciente de 39 años, sin antecedentes médicos relevantes, quien cursa con su tercera gestación de 37 semanas. Consulta al hospital de referencia local por contracciones uterinas regulares dolorosas y sensa- ción de pujo. No había asistido a control prenatal. Presentó ruptura espontánea de membranas una hora antes. Al examen físico realizado por el médico de turno evidencia trabajo de parto en periodo expulsivo, borramiento del $100 \%$ y dilatación de $10 \mathrm{~cm}$, feto en presentación de pelvis, modalidad completa, con expulsión de meconio grado I y estación +2 . Se ordena traslado inmediato a sala de cirugía para cesárea de emergencia y valoración por el obstetra. Se alerta a los servicios de pediatría y anestesiología. Sin embargo, un par de minutos luego de su valoración, la paciente incrementa el pujo y se aprecian partes fetales en el introito: el parto vaginal es inminente.

\section{REVISIÓN DE LA LITERATURA}

\section{Elementos de la semiología obstétrica en presentación pelviana}

El diagnóstico del trabajo de parto con feto en presentación pelviana se realiza mediante la palpación del abdomen materno (maniobras de Leopold) con el objeto de establecer la presentación, posición y actitud fetal, tratando de sospechar la presencia de extensión cefálica, que pudiera determinar el riesgo de retención de la cabeza última $(6,20,21)$. El clínico no debe olvidar que la auscultación de la fetocardia en la parte superior del abdomen materno también representa un indicio que permite sospechar esta presentación $(1,2)$.

En el contexto del trabajo de parto establecido, el profesional debe identificar el punto de reparo de la presentación mediante el tacto vaginal (6, 20-22). Si la dilatación cervical es suficiente, se palpa una superficie irregular, blanda, acompañada de prominencias y depresiones. Masa separada en dos, por la presencia de un surco más o menos pronunciado (surco interglúteo) y en cuya mitad se encuentra una pequeña depresión que corresponde al ano $(22,23)$.

En uno de los extremos de dicho surco es factible identificar la presencia de una pequeña prominencia ósea triangular, ligeramente flexible, que corresponde al coxis y, por encima de este, tres 
o cuatro prominencias óseas que corresponden a la cresta sacra, punto de reparo de la presentación. La prominencia sacrocoxígea es esencial para el diagnóstico de la presentación y casi siempre se reconoce con relativa facilidad $(22,23)$.

Para poder diferenciar con el tacto entre un pie y la mano, el profesional debe recordar que el pie se reconoce por la existencia de tres prominencias óseas (maléolos y talón), el ángulo a nivel del calcáneo y la disposición de los dedos: cortos y colocados sobre la misma línea, sin oposición del pulgar a los restantes. Finalmente, es clave recordar que el surco interglúteo representa, desde el punto de vista de la variedad de posición, el mismo papel que la sutura sagital para la presentación de vértice (23), siendo frecuente que se perciba algún grado de "asinclitismo" glúteo.

\section{Mecanismos del trabajo de parto en presentación pelviana}

En la presentación pelviana hay tres pasos sucesivos: a) el desprendimiento pélvico, b) el desprendimiento de los hombros y c) el desprendimiento cefálico. Cada uno de estos segmentos se desprende con dificultad progresiva dado que ostenta un diámetro cada vez mayor: el bitrocantéreo menor que el biacromial y este menor que el suboccipitofrontal $(23,24)$. León, en un texto clásico de Obstetricia, describió los diez tiempos del parto en pelvis (23); se señalan a continuación, conjuntamente con las recomendaciones del profesor Peralta Cayón (24).

- Primer tiempo: corresponde a la acomodación de las nalgas en el estrecho superior (23) y se produce gracias a que el feto reduce sus diámetros por apelotonamiento y compresión. Este aminoramiento ocurre al unísono con la orientación hacia un diámetro oblicuo del diámetro bitrocantéreo, el cual posee una dimensión relativamente fija de 9,5 centímetros, y estará encargado de presidir el parto (24).

- Segundo tiempo: demarca el encajamiento y descenso del polo pelviano. Se lleva a cabo por simple progresión en sentido oblicuo con ligero asinclitismo posterior; esto se verifica porque la nalga posterior desciende más abajo que la anterior y el surco interglúteo está más cerca del pubis que del sacro (23). El encajamiento culmina cuando se alcanza el estrecho inferior. Este tiempo es laborioso en la modalidad de pelvis completa y versátil en la incompleta (24). - Tercer tiempo: corresponde a la acomodación del polo pélvico fetal en el estrecho inferior de la pelvis materna. El encajamiento de la presentación ocurre generalmente en una variedad de posición oblicua; se encuentran cuatro posibilidades: sacra izquierda anterior, sacra derecha anterior (SIA y SDA respectivamente), sacra izquierda posterior, sacra derecha posterior (SIP y SDP respectivamente). Una vez el polo pélvico fetal hace contacto con el estrecho inferior ocurre una rotación interna de 45 grados. En las variedades anteriores ocurre una rotación en 1/8 de círculo hacia atrás, en las variedades posteriores se presenta una rotación igual pero hacia adelante. Esta es la primera rotación de las cuatro clásicas del parto en pelvis. Las rotaciones internas se refieren a mecanismos realizados en el canal del parto y las rotaciones externas a mecanismos de las partes fetales por fuera del canal del parto (23).

- Cuarto tiempo: señala el desprendimiento del polo pelviano (figura 1). Así, el polo pélvico se va a desprender en sacro transversa, el diámetro bitrocantéreo coincide con el diámetro anteroposterior del estrecho inferior: subpubiano-sacro y, de esta forma, el surco interglúteo se orienta transversalmente. La nalga anterior, ubicada bajo el pubis, entreabre el orificio vulvar, en tanto que su homónima posterior lleva el coxis hacia atrás, abomba el periné y se exterioriza; solo entonces se desprende enteramente la nalga anterior. A esta altura del expulsivo, el cuerpo fetal ha presentado una inflexión lateral, describiendo una curvatura cuya concavidad apunta hacia el pubis. Habitualmente, cuando se trata de una 


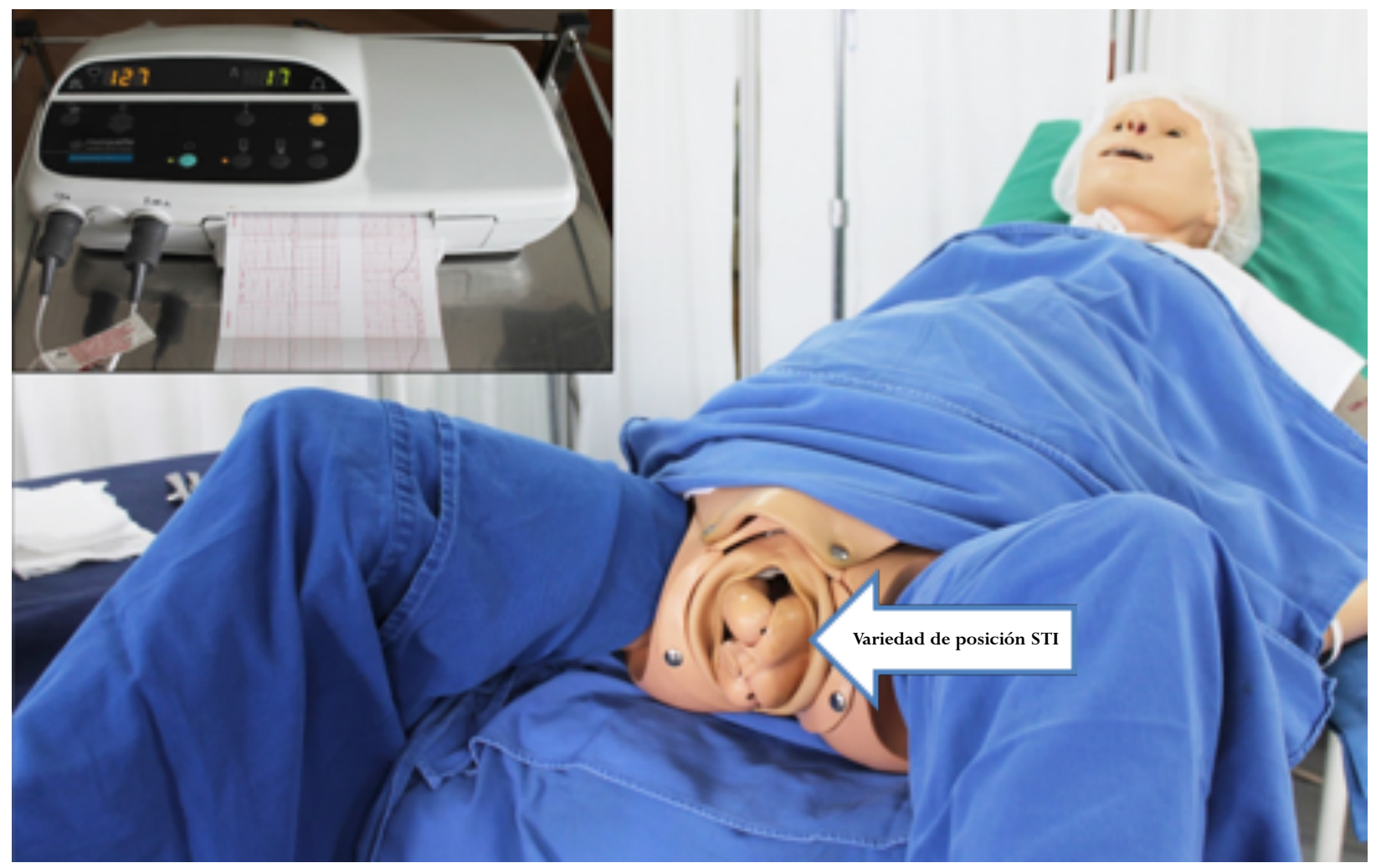

Figura 1. Desprendimiento del polo pélvico fetal. Variedad de posición sacro transverso izquierdo; el surco interglúteo se orienta transversalmente (flecha)

presentación en pelvis completa, las extremidades inferiores se expulsan de manera simultánea con las nalgas (23).

- Quinto tiempo: corresponde a la acomodación de los hombros en el estrecho superior. Las extremidades superiores presentan flexión forzada reduciendo el diámetro biacromial para luego acomodarse al diámetro oblicuo por el cual pasó el bitrocantéreo si se trata de una variedad anterior. Para las variedades posteriores, el diámetro bitrocantéreo coincide con el diámetro oblicuo opuesto. La orientación oblicua de los hombros explica por qué se produce la rotación externa del tronco fetal (restitución) desde una orientación oblicua hacia adelante. El dorso fetal se torna subpúbico. En este tiempo ocurre la segunda rotación de 90 grados que tiene por objeto colocar al feto con el raquis en posición anterior bajo la sínfisis (23).
- Sexto tiempo: demarca el encajamiento y descenso de los hombros. Para entonces, se ha producido el desprendimiento del abdomen y de la porción más inferior del tórax fetal (23).

- Séptimo tiempo: señala la acomodación de los hombros en el estrecho inferior al experimentar una rotación interna de 45 grados, de tal forma que el diámetro biacromial coincide con el subsacro subpubiano. Esta corresponde a la tercera rotación, donde el diámetro biacromial se torna anteroposterior a nivel del estrecho inferior y el dorso fetal queda dirigido hacia el lado derecho o izquierdo de la madre (4). Durante este tiempo, la cabeza se acomoda en el estrecho superior mediante la flexión, y orientando su diámetro mayor al diámetro oblicuo opuesto al que utilizó el biacromial. No obstante, la flexión no es forzada de tal forma que, el diámetro occipito-mentoniano es sustituido por el diámetro suboccipito-frontal (23). 
- Octavo tiempo: demarca el desprendimiento de los hombros. El hombro anterior se calza bajo la sínfisis púbica a la altura del acromion y el posterior empuja el coxis hacia atrás. De esta forma, el hombro posterior se desprende gracias a un movimiento de inflexión lateral en sentido antero-superior de forma similar al que se realizó para el desprendimiento de la pelvis fetal. Pronto, el hombro anterior se desprende también por un movimiento de inflexión lateral, pero en esta ocasión en sentido antero-inferior. Para entonces, la cabeza ha descendido por el diámetro oblicuo a través de los diferentes estrechos alcanzando el inferior (23).

- Noveno tiempo: se refiere a la acomodación de la cabeza en el estrecho inferior, la cual, totalmente flejada, experimenta un movimiento de rotación interna para que el diámetro suboccipito-frontal se ponga en relación con el diámetro subsacro subpubiano; es la cuarta rotación y persigue colocar el occipucio bajo la sínfisis. No obstante, si el mentón se torna anterior, aparece una distocia rotacional difícil de solucionar (3). Este conjunto de movimientos se observa desde el exterior como la rotación externa de los hombros en donde el dorso fetal se direcciona hacia arriba (23).

- Décimo tiempo: finalmente, el desprendimiento de la cabeza. El occipucio localizado en el espacio retropúbico, sirve como punto fijo en torno al cual la cabeza ejecuta un movimiento de flexión y progresión. El mentón, la boca, la nariz y la frente aparecen paulatinamente por la horquilla para finalmente salir el occipucio bajo la sínfisis del pubis (23).

El profesor Peralta Cayón, en un trabajo realizado en 1940, informaba que la expulsión espontánea del feto es la regla en el $69 \%$ de los casos (24). Debemos resaltar, entonces, que el parto en pelvis tiene cuatro diferencias muy significativas con respecto al parto cefálico de vértice: el encajamiento ocurre en el estrecho inferior en lugar del medio; los tiempos son diez comparados con los seis del vértice; las rotaciones son cuatro comparadas con dos en vértice, y la evolución espontánea ocurre con menor frecuencia que para el vértice $(97 \%)(2,24)$.

\section{Manejo intraparto de la gestante con feto en presentación pelviana}

El parto en presentación pelviana debe ser atendido por vía vaginal cuando se encuentra en periodo expulsivo y el sacro fetal ha traspasado el estrecho inferior de la pelvis, debido a que los riesgos de su atención por cesárea superan los beneficios $(5,18)$. Para atender el parto en presentación de pelvis se ha descrito que es conveniente contar por un equipo constituido por: un profesional encargado de la atención del parto, otro de la atención neonatal; además, una persona que asista las maniobras y, finalmente, alguien encargado de la provisión de suministros $(3,25)$. La gestante debe tener un acceso venoso permeable y se debe realizar hemoclasificación ante el riesgo de hemorragia posparto $(21,22)$.

En ausencia de estudios conclusivos, la posición materna puede ser de litotomía o vertical (5, 6, 26) y, dado que no existe evidencia suficiente, el refuerzo del trabajo de parto con oxitocina $(5,6)$ puede ser una alternativa por considerar cuando la dinámica uterina no es apropiada $(1,5,18,25)$. El Maternal Fetal Medicine Committee (MFMC) recomienda evitar la amniorrexis, ofrecer una alternativa para el manejo del dolor, efectuar vigilancia del bienestar fetal (25), así como de la adecuada progresión del parto $(5,24)$.

Por otra parte, se ha descrito que la episiotomía debe ser restrictiva $(1,5)$ y si se identifica que el cordón se encuentra a tensión es aconsejable realizar un asa del mismo (15). El MFMC recomienda no realizar extracción, ni tracción del cuerpo fetal (5, 25) (figura 2) ya que la tracción de los miembros inferiores del feto o del tronco deshace el cilindro fetal y puede redundar en deflexión de la cabeza o extensión nucal de los brazos. También, recomienda preparar compresas tibias para envolver 
los miembros inferiores del feto (25); asimismo, se recomienda mantener una serena tranquilidad y una espera inteligente, reservando las maniobras solo en caso de ser necesario (5). Por último, se recomienda hacer pinzamiento diferido del cordón en la presentación de pelvis $(27,28)$.

\section{Maniobras obstétricas aplicables en el parto de pelvis}

Las fuerzas naturales del parto se dejan actuar libremente hasta que se visualiza el ángulo inferior del omoplato fetal. En este instante, la cabeza penetra en la pelvis menor, comprime el cordón y dificulta la oxigenación por lo que surge el riesgo de asfixia; no obstante, el obstetra dispone de cuatro minutos para terminar el desprendimiento fetal (29-31). Pese a esta aparente premura, el obstetra debe recordar el predicamento señalado por Stockel, citado por Schwarcz: "En la presentación pelviana el ritmo de salida fetal debía ser lento hasta la salida del ángulo del omoplato, luego rápido hasta que la boca atravesaba la vulva y de nuevo lento hasta completar la expulsión de la cabeza" (32). Esta última lentificación del proceso pretende evitar lesiones fetales y desgarros del canal del parto (32).

Las maniobras se clasifican en: aquellas que facilitan el desprendimiento de los brazos y la cabeza (v. g. maniobra de Bracht), de los hombros (v. g. Pajot, Rojas-Lowset), y la cabeza última (v. g. Mauriceau, Praga) (19, 29, 31).

Maniobra de Bracht: fue descrita en 1935 por Erich Bracht, obstetra alemán. Es la única que tiene por objeto facilitar el desprendimiento simultáneo de los hombros y la cabeza $(30,33)$, inicia cuando el ángulo inferior de las escápulas fetales aparece en la vulva, y una vez ha ocurrido la segunda rotación y el dorso fetal se orienta hacia adelante. Entonces, se empalman con ambas manos los muslos y el tronco fetal (los pulgares presionan los muslos flexionados sobre el abdomen y los cuatro dedos restantes de cada mano se aplican sobre la región lumbosacra) levantándolos suavemente sin traccionar, con lo que se logra el desprendimiento de los brazos (30, 33, 34). Posteriormente, el obstetra aproxima las nalgas

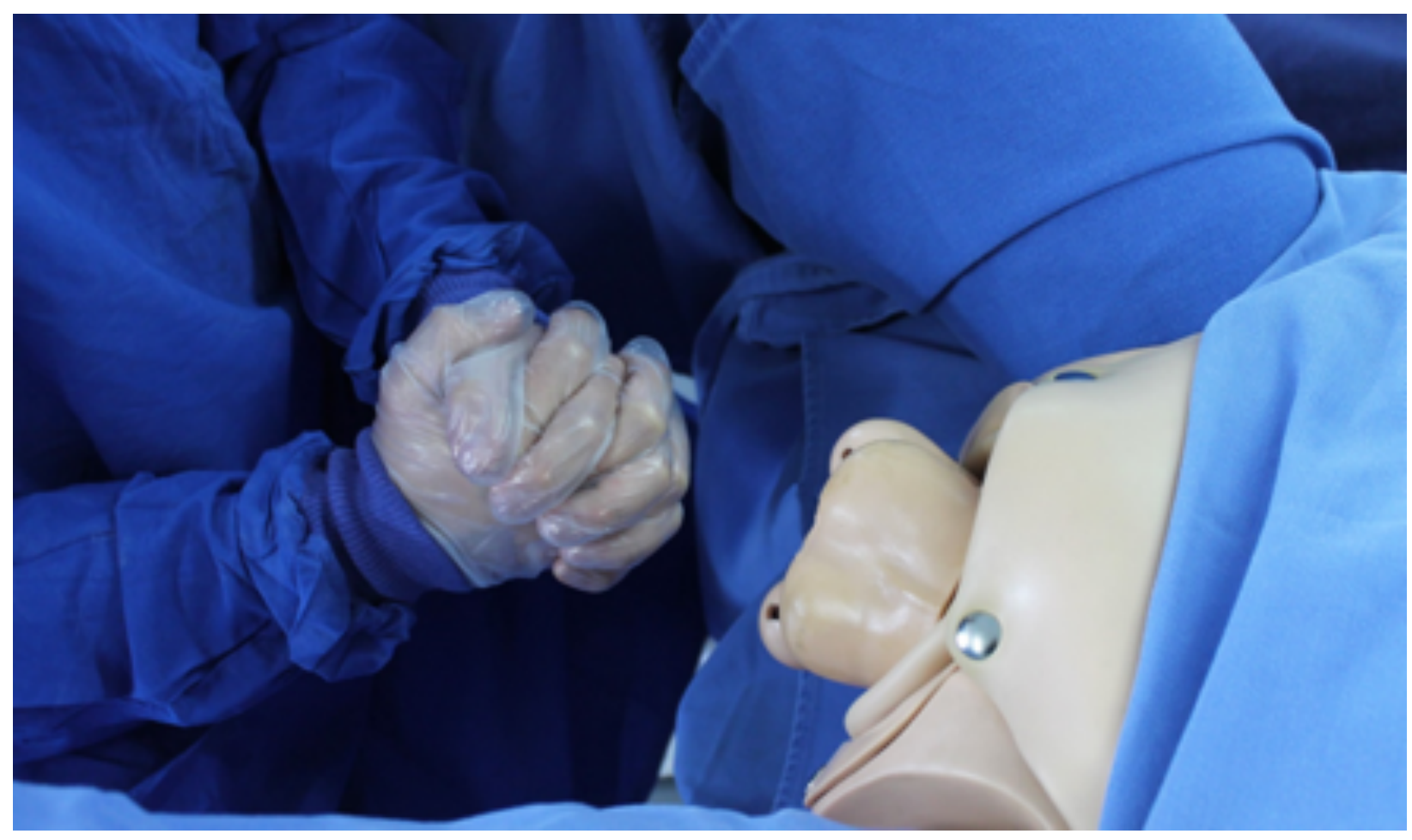

Figura 2. Evitar la extracción y tracción del cuerpo fetal durante el trabajo de parto 
fetales y el dorso del feto al hipogastrio materno, con lo que se logra el desprendimiento fetal $(30,33)$.

La escuela uruguaya considera esta maniobra como de primera línea, pues soluciona la detención de parto cuando la cintura escapular ha llegado al plano de la vulva. Consideración que probablemente se fundamenta en que soluciona el 60\% de los partos vaginales con presentación en pelvis (32).

Bratch decía: "El arte de la espera es difícil y no hay muchos obstetras que tengan ya sea el valor o la paciencia para quedarse cruzados de brazos mientras los partos de nalgas se producen de forma espontánea. Esto se hace aún más difícil si el obstetra impaciente tiene un siglo de tradición, así como las palabras y los escritos de los maestros contemporáneos detrás de él" (35). Es así, como el célebre profesor de Obstetricia Juan León, llamó a Bracht el "campeón de la espera” al sentar el precepto fundamental para la atención de este tipo de partos (23).

Maniobra de Rojas: descrita por el profesor argentino Daniel Alberto Rojas en 1930 (29), se realiza tomando el feto por los muslos apoyando los pulgares sobre el sacro, obligándolo a rotar sobre su plano ventral de tal modo que el hombro que primitivamente era posterior se transforma en anterior (figura 3), al tiempo que el brazo correspondiente se moviliza tal y como lo revela la aparición del ángulo inferior de la escápula, cuyo resalto aparece por debajo del pubis. Con ello, el obstetra puede tomar finalmente el codo y desprender el brazo. Corresponde ahora "desandar lo andado"; se toma nuevamente el sacro fetal volviendo a llevar el hombro que ahora es posterior a posición anterior contrarrotando el feto alrededor de su eje con lo que ocurre el desprendimiento del segundo (29). Esta maniobra también es conocida en la escuela escandinava con el nombre de maniobra de Lovset, publicación que data de 1937 (36).

Maniobra de Pajot: descrita por Charles Pajot, profesor de Obstetricia de la Universidad de París (9). Se levanta el cuerpo fetal y se desliza la mano entre el canal del parto y el hombro posterior alcanzando el codo, para lograr la extracción de la extremidad al realizar un movimiento en el que el brazo pasa por delante de la cara (figura 4). Posteriormente, se extrae el brazo anterior realizando un procedimiento similar (9).

Maniobra de Praga: descrita por Prezos en 1573, pero introducida en la práctica en 1846 por Kiwisch (29), se realiza posterior al desprendimiento de

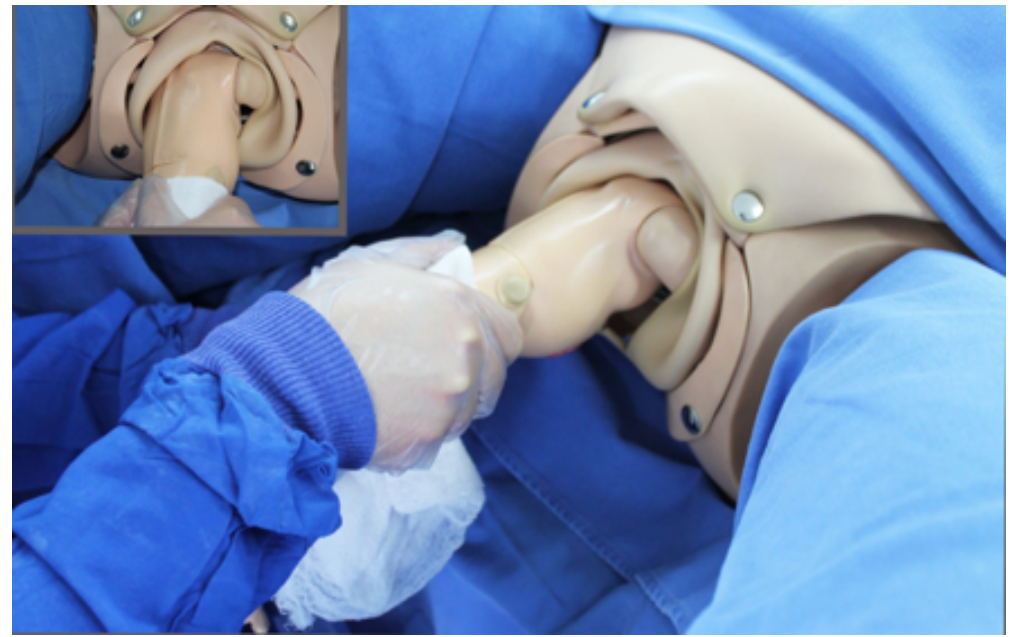

Figura 3. Maniobra de Rojas

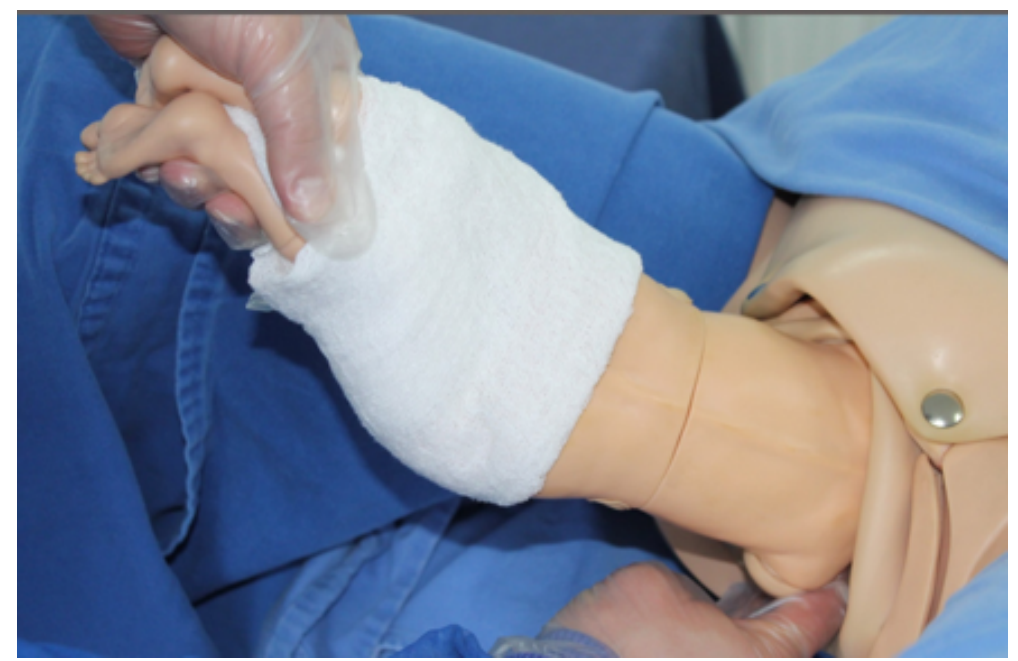

Figura 4. Maniobra de Pajot 
los hombros, cuando la cabeza fetal está encajada (30). Se toman con la mano derecha los miembros inferiores del fruto en tanto que la mano izquierda apoya los dedos índice y medio en forma de horquilla sobre la nuca (figura 5) $(31,36)$. Se ejerce con ambas manos una tracción hacia abajo para conducir el occipucio bajo la sínfisis (31), para entonces levantar el cuerpo fetal colocando el dorso de este sobre el vientre materno. Para el éxito de esta maniobra se debe detener la tracción hacia abajo en cuanto el occipucio se coloca bajo la sínfisis. Puede ser útil la presencia de un ayudante que ejerza presión sobre la cabeza fetal desde el abdomen. La maniobra puede efectuarse también con la cabeza fetal en occipitosacra, siguiendo una técnica similar llamada maniobra de Praga invertida $(31,36)$.

Maniobra de Mauriceau-Smellie-Veit: descrita por Mauriceau en 1668, redescubierta por Smellie e incorporada definitivamente por Veit en 1863 en la escuela de Viena, tiene como objeto flejar la cabeza fetal acomodándola al estrecho inferior con miras a facilitar su desprendimiento, se realiza cuando la cabeza se encuentra encajada ya sea en posición

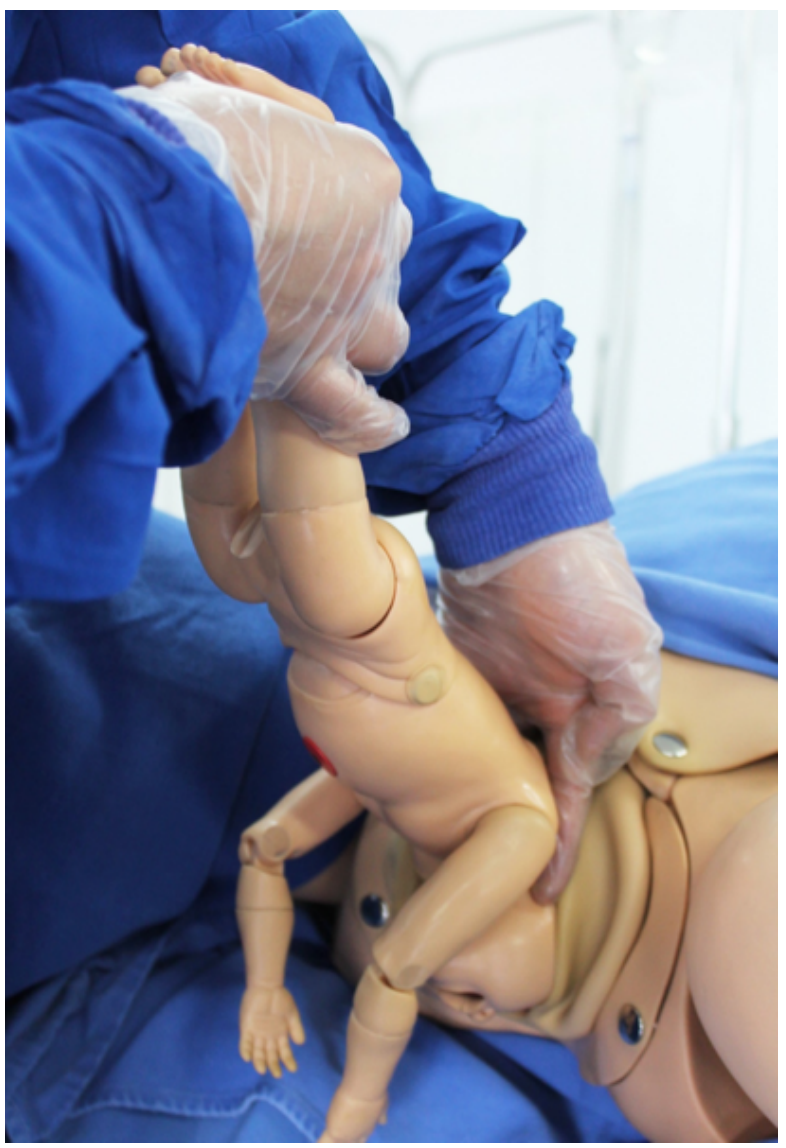

Figura 5. Maniobra de Praga occipito-púbica, oblicua anterior o transversa (30). Se ejecuta de la siguiente manera: se hace cabalgar al cuerpo fetal sobre el antebrazo, que corresponde a la mano que se introducirá en la vagina; por su parte, el dedo índice y medio de esta extremidad, orientados en sentido palmar, se dirigen por el plano ventral del feto en busca de la boca de este (figura 6) para tomar apoyo sobre la base de la lengua evitando enganchar el maxilar inferior o el piso de la boca, pues pueden ocurrir accidentes fetales $(30,31,33)$.

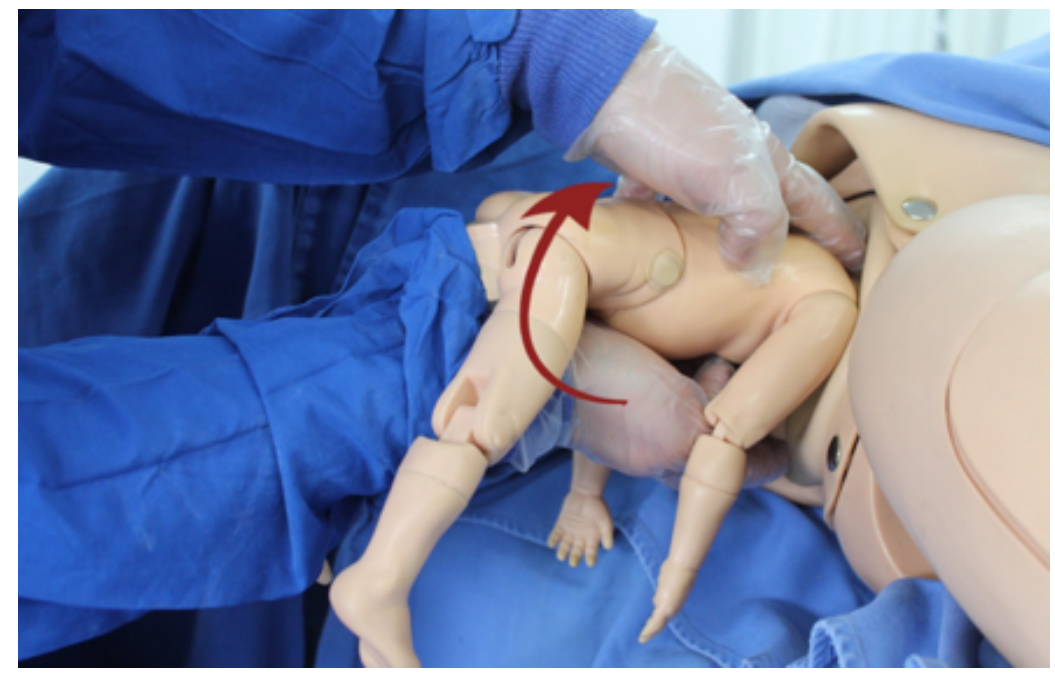

Figura 6. Maniobra de Mauriceau-Smellie-Veit

Por estos potenciales accidentes, algunos autores prefieren realizar la presión sobre la cara fetal por fuera de la boca, a nivel de las eminencias malares o el maxilar superior (37).
Por otra parte, el dedo índice y medio de la otra mano cuya palma se dirige en el plano dorsal del fruto, se posiciona en forma de "tenedor" alrededor del cuello. Las extremidades de los dedos deben 
reposar en la medida de lo posible sobre el esternón y no sobre la cara lateral del cuello, evitando colocar los dedos en forma de gancho para no lesionar el paquete vásculo-nervio-supraclavicular $(30,31)$. A continuación, las manos actúan simultáneamente aunque desempeñan un papel distinto para: maximizar la flexión de la cabeza fetal por parte de los dedos introducidos en la boca (mentón en contacto con el esternón); rotar la cabeza fetal hasta que el occipucio se localice en la cara posterior del pubis llevando la cara fetal a la concavidad del sacro (30, 31) y desprender la cabeza fetal bien flexionada y orientada en el diámetro antero-posterior del estrecho inferior, ejerciendo cierta tracción hacia abajo hasta que el occipucio aparezca bajo la sínfisis pubiana $(30,31)$. Esta tracción es ejercida por la mano colocada sobre los hombros y no por aquella que se encuentra en la boca del fruto (31). Finalmente, solo cuando el occipucio aparece, se levanta el cuerpo fetal con el antebrazo que lo soporta en dirección al abdomen materno manteniendo flexionada la cabeza $(30,31)$.

Se han descrito algunas variantes a esta maniobra clásica y fundamental. La maniobra de Arnot (utilizada de manera preferencial por uno de los autores), en la cual, la flexión de la cabeza se realiza mediante el apoyo de los dedos sobre el maxilar superior a lado y lado de la nariz, evitando la salida de los nervios infraorbitarios, y la maniobra de MuñozArbat, en donde el dedo medio de la mano que se apoya en la nuca fetal, presiona sobre el occipucio y flexiona la cabeza (37).

Finalmente, es importante que el clínico conozca y domine las opciones de manejo para el abordaje del parto obstruido por atrapamiento de la cabeza última. En este sentido, el desprendimiento mediante fórceps especialmente diseñados (Piper, 1929), la sinfisiotomía, la cesárea de emergencia con restitución fetal a la cavidad uterina (maniobra de Zavanelly) y las incisiones de Dührssen (se realizan en las horas 2, 6 y 10, dibujando una clásica "Y" a nivel cervical) cuando la obstrucción corresponde a un cuello edematoso y rígido, representan las alternativas finales para el manejo de esta condición $(1,5,19)$. Si el desprendimiento de los hombros ya ha ocurrido de manera espontánea o mediante maniobras obstétricas propias, como la maniobra de Rojas, se debe recurrir a la maniobra de Mauriceau; si esta fracasa es urgente recurrir prontamente al fórceps de Piper (32). Recurrir a la fuerza en la aplicación de la maniobra de Mauriceau podría ser contraproducente para el feto. Recordemos el postulado de Doderlein, citado por Schwarcz: "la aplicación del fórceps a la cabeza última conservaba más niños vivos que las maniobras" (32).

\section{CONCLUSIONES}

El mecanismo del parto en presentación pelviana es complejo y requiere, cuando no hay otra alternativa para la atención, que tanto el obstetra como el médico general conozcan su fisiología y las múltiples maniobras obstétricas que facilitan obtener un buen resultado materno-perinatal.

\section{AGRADECIMIENTOS}

Agradecemos al doctor Hernando Gaitán-Duarte, por facilitar a los autores libros clásicos de la obstetricia.

\section{REFERENCIAS}

1. The Royal Women`s Hospital, Victoria Australia. Management of breech presentation, Clinical Guideline. 2012. Disponible en: https://www. thewomens.org.au/health-professionals/clinicalresources/clinical-guidelines-gps/ .

2. Ñáñez H, Ruiz A, Ángel E, Bautista A, Sabogal J. Distocias. En: Ñánez H, Ruiz A (eds.). Texto de obstetricia y perinatología. Bogotá: Lito Camargo; 1999. p. 105-121.

3. Botella-Llusia J, Clavero-Núnez J. Distocias de los elementos del parto. Presentación podálica. Clasificación y nomenclatura. En: Tratado de ginecología, Tomo II, Patología obstétrica. 12 ed. Barcelona, Editorial Científica Médica; 1981. p. 575-599. 
4. Arias F, editor. Abnormal Labor and delivery. En: Practical Guide to High-risk Pregnancy. 2 ed. Mosby Year Book; 1993. p. 402.

5. Royal College of Obstetricians \& Gynaecologists. Management of Breech Presentation, Clinical Guideline. UK 2017. Disponible en: https://www.rcog. org.uk/en/guidelines-research-services/guidelines/ gtg20b/.

6. The Royal Australian and New Zealand College of Obstetricians and Gynecologists. Management of Breech Presentation at Term, Clinical Guideline. 2016. Disponible en: https://www.ranzcog.edu.au/RANZCOG_SITE/ media/RANZCOG-MEDIA/Women \%27s\%20Health/ Statement\%20and\%20guidelines/Clinical-Obstetrics/ Management-of-breech-presentation-at-term-(CObs-11)-Review-July-2016pdfBreechPresentationInTheAntenatalPeriodAndBreechDeliveryClinicalGuidelinesForTheManagementOf.pdf.

7. Royal Cornwall Hospital. Clinical Guideline for the Management of a Breech Presentation in the Ante Natal Period, External Cephalic Version (ECV) and Breech Presentation in Labour, UK 20212. Disponible en: http://www.rcht.nhs.uk/ DocumentsLibrary/RoyalCornwallHospitalsTrust/ Clinical/MidwiferyAndObstetricsBreechPresentationInTheAntenatalPeriodAndBreechDeliveryClinicalGuidelinesForTheManagementOf.pdf.

8. Fianu S, Václavínková V. The site of placental attachment as a factor in the aetiology of breech presentation. Acta Obstet Gynecol Scand. 1978;57(4):371-2. https://doi. org/10.3109/00016347809154033

9. Cabero-Roura L. Patología del parto (III): Presentaciones anormales. El parto de nalgas. Conducta obstétrica. En: Cabero-Roura L, Saldivar-Rodríguez D, Cabrill-Rodríguez E. Obstetricia y medicina Maternofetal. Madrid: Editorial Médica Panamericana; 2007. p. 994.

10. Duffy CR, Moore JL, Saleem S, Tshefu A, Bose CL, Chomba E, et al. Malpresentation in low- and middleincome countries: Associations with perinatal and maternal outcomes in the Global Network. Acta
Obstet Gynecol Scand. 2019; 98(3):300-8. https:// doi.org/10.1111/aogs.13502.

11. Kozuki N, Katz J, Khatry SK, Tielsch JM, LeClerq SC, Mullany LC. Risk and burden of adverse intrapartumrelated outcomes associated with non-cephalic and multiple birth in rural Nepal: A prospective cohort study. BMJ Open. 2017;7(4):e013099. http://dx.doi. org/10.1136/bmjopen-2016-013099

12. Dohbit JS, Foumane P, Tochie JN, Mamoudou F, Temgoua MN, Tankeu R, et al. Maternal and neonatal outcomes of vaginal breech delivery for singleton term pregnancies in a carefully selected Cameroonian population: A cohort study. BMJ Open. 2017;7(11):e017198. https://doi.org/10.1136/ bmjopen-2017-017198.

13. Fonseca A, Silva R, Rato I, Neves AR, Peixoto C, Ferraz Z, et al. Breech presentation: Vaginal versus cesarean delivery, which intervention leads to the best outcomes? Acta Med Port. 2017;30(6):479-84. https://doi.org/10.20344/amp.7920.

14. Lai S, Flatley C, Kumar S. Perinatal risk factors for low and moderate Five-minute Apgar scores at term. Eur J Obstet Gynecol Reprod Biol. 2017;210:251-6. https://doi.org/10.1016/j.ejogrb.2017.01.008.

15. Mid Essex Hospital Services. Management of breech birth 4.0. UK 2014. Disponible en: http://www.meht. nhs.uk/search/?q=breech.

16. Yeoh SGJ, Rolnik DL, Regan JA, Lee PYA. Experience and confidence in vaginal breech and twin deliveries among obstetric trainees and new specialists in Australia and New Zealand. Aust N Z J Obstet Gynaecol. 2019;59(4):545-9. https://doi.org/10.1111/ ajo. 12931.

17. Petrovska K, Sheehan A, Homer CSE. The fact and the fiction: A prospective study of internet forum discussions on vaginal breech birth. Women Birth. 2017;30(2):e96-e102. https://doi.org/10.1016/j. wombi.2016.09.012.

18. ACOG Committee Opinion No. 745. Mode of term singleton breech delivery. Obstet Gynecol. 2018;e60-E63. Doi: https://doi.org/10.1097/AOG. 0000000000002755 
19. Hofmeyr GJ, Kulier R. External cephalic version for breech presentation atterm. Cochrane Database Syst Rev. 2012;10:CD000083. https://doi. org/10.1002/14651858.CD000083.pub2.

20. Auckland District Health Board, National Women`s Hospital, Auckland New Zealand. Breech birth. Clinical Guideline. 2012. Disponible en: http://nationalwomenshealth.adhb.govt.nz/healthprofessionals/ policies-and-guidelines

21. Nottingham University Hospital. Guideline for the management of singleton breech presentation in pregnancy and labour. UK 2012. Disponible en: https://www.nuh.nhs.uk/download.cfm?doc $=$ docm93jijm4n1050.pdf\&ver $=11159$

22. East Cheshire Hospital. Breech presentation. Clinical Guideline. UK 2013. Disponible en: http://www. eastcheshire.nhs.uk/About-The Trust/policies/M/ Maternity\%20Breech \%20Presentation \%201907.pdf

23. León J. El parto en presentación pelviana. En: Tratado de Obstetricia. Tomo I. Buenos Aires: Editorial Científica Argentina; 1956. p. 1066-1115.

24. Peralta Cayón R. Presentación de pelvis y de hombros. En: Obstetricia y Clínica Obstétrica. Bogotá: Imprenta Antares; 1956. P. 697-753.

25. Kotaska A, Menticoglou S, Gagnon R. Maternal Fetal Medicine Committee. Vaginal delivery of breech presentation. J Obstet Gynaecol Can. 2009;31(6):557-66. https://doi.org/10.1016/S17012163(16)34221-9

26. Louwen F, Daviss BA, Johnson KC, Reitter A. Does breech delivery in an upright position instead of on the back improve outcomes and avoid cesareans? Int J Gynaecol Obstet. 2017;136(2):151-61. https://doi. org/10.1002/ijgo.12033.

27. Rabe H, Diaz-Rossello JL, Duley L, Dowswell T. Effect of timing of umbilical cord clamping and other strategies to influence placental transfusion at preterm birth on maternal and infant outcomes. Cochrane
Database of Systematic Reviews. 2012; Issue 8. Art. CD003248. https://doi.org/10.1002/14651858. CD003248.pub3.

28. Currea-Guerrero S. La adaptación neonatal inmediata: la reanimación neonatal. Bogotá: Universidad Nacional de Colombia; 2004.

29. Muñoz-Arbat J. Extracción manual del feto y versión. En: Nubiola P, Zárate E, editores. Tratado de Obstetricia. Barcelona: Editorial Labor; 1951. p. 963-1020.

30. Lee J, Greenhill JP, Carrera O, Peralta A, Carrasco R. Anormalidades por parte del feto presentación de nalgas. En: Principios y práctica de obstetricia. Tomo II. México: Unión Tipográfica - Editorial Hispano Americana; 1947. p. 790-830.

31. Dexus Trias de Bes S, Carrera Macia JM. Parto podálico operatorio. En: Tratado de Obstetricia Dexeus. Volumen III: Tratado y atlas de Operatoria Obstétrica. 2 ed. Barcelona: Salvat Editores; 1988.

32. Schwarcz RL, Duverges CA, Diaz G, Fescina RH. Obstetricia. El parto en las distintas presentaciones. 5 ed. Buenos Aires: El Ateneo; 1997. p. 450.

33. UMVF - Université Médicale Virtuelle Francophone. Manœuvres obstétricales. Comité éditorial de l'UVMaF 2013-2014. p. 4-29.

34. Feliz Báez CA. Presentación pélvica. Manual de maniobras y procedimientos en Obstetricia. Bogotá: McGraw-Hill; 2005. p. 235-245.

35. Dunn PM. Erich Bracht (1882-1969) of Berlin and his "breech" manoeuvre. Arch Dis Child Fetal Neonatal Ed. 2003;88(1):F76-7. https://doi.org/10.1136/ fn.88.1.f76

36. Lovset J. Shoulder delivery by breech presentation. J Obstete Gynaecol. 1973;44:696-704. https://doi. org/10.1111/j.1471-0528.1937.tb14687.x

37. Pschyrembel W. Presentación de la extremidad pélvica. En: Obstetricia práctica. 3 ed. Buenos Aires: Editorial Labor; 1978. p. 135-164. 


\section{CONTRIBUCIÓN DE LOS AUTORES}

Carlos Fernando Grillo-Ardila: elaboración del documento desde su concepción y diseño hasta la adquisición de la información, revisión del contenido intelectual y aprobación de la versión enviada a proceso editorial, elaboración de fotos y participación en el diseño del material gráfico.
Alejandro Antonio Bautista-Charry: elaboración del documento desde su concepción y diseño hasta la adquisición de la información, revisión del contenido intelectual y aprobación de la versión enviada a proceso editorial.

Mariana Diosa-Restrepo: elaboración del documento desde su concepción y diseño hasta la adquisición de la información, revisión del contenido intelectual y aprobación de la versión enviada a proceso editorial. 